\title{
Useful Oblivion Versus Information Overload in e-Learning Examples in the Context of Wiki Systems
}

\author{
Christian Stickel ${ }^{1}$, Martin Ebner ${ }^{1}$ and Andreas Holzinger ${ }^{2}$ \\ ${ }^{1}$ Department for Social Learning, Graz University of Technology, Austria \\ ${ }^{2}$ Research Unit HCI4MED, Institute of Medical Informatics, Statistics and Documentation, Medical University Graz, Austria
}

\begin{abstract}
Information overload refers to the state of having too much information to make a decision or remain informed about a topic. We present a novel approach of filtering, adapting and visualizing content inside a Wiki knowledge base. Thereby we follow the question of how to optimize the process of learning, with respect to shorter time and higher quality, in face of increasing and changing information. Our work adopts a consolidation mechanism of the human memory, in order to reveal and shape key structures of a Wiki hypergraph. Our hypothesis so far is that visualization of these structures enables a more efficient learning.
\end{abstract}

Keywords: e-learning, neural networks, wiki, information visualization, mental models

\section{Introduction}

One of the most frequently asked questions in our modern information-based lifelong learning society surely is: How can we optimize the process of learning, within shorter time and growing availability of information?

A wide spectrum of e-learning technologies has been developed, including various authoring systems, virtual simulations, digital learning games, mobile technologies [14] etc.; ranging from simple information presentation to highly multimedia-based and interactive applications [15]. However, most of those technologies are designed to present information to the learner, of course, at any time and any place. Following the discussions on Web 2.0, it becomes obvious that the presentation of information is predominant [6]; at least such technology enables authoring of everyone - and the end users can customize both content and form [17].

If applied appropriately in an e-learning setting, Wiki systems can combine the concept of explorative learning (tools and information are provided and the learner makes "sense") in a hypermedia system with collaborative interactive construction of knowledge (every user can change the content). As the method of explorative learning also implies learning without a rigid learning path, it gives the responsibility and the power to the users [12]. However, the novice learner without a basic mental model in the field of knowledge might get lost in hyperspace and overloaded with information, when presented a large multi-linear learning object such as a Wiki knowledge base. The main problem here is an adequate navigation and visualization of the content in order to optimally support the cognitive performance of the users [12].

Based on these observations, we propose an approach, which supports the learning efforts by automatically generating transformations of the structures (local Webgraph; network) on the one hand and providing a metastructure overview on the other. This is done with a vote algorithm [16], an elementary memory process, which consolidates information by aggregation and disaggregation [11] and a hyperbolic tree to visualize a navigable metastructure of the existing knowledge inside the system. The metastructure is thereby meant to show the most relevant links (edges) and the most relevant documents (nodes). It is built automatically, 
while users are browsing and editing the content. With every action of a user the system itself learns which nodes are most important and which edges are used most. The important difference from other systems and approaches e.g. [4][5] is that links (edges) can be "forgotten" when not used. In this process, a complex structure is consolidated.

We assume that this enables the learner to gain insight into the crucial key points and the main structure of the changing knowledge inside the system. At the same time the user gets feedback on his progress and the progress of the group, as the reorganization and recategorization of the contents is always visible to him. We also intend to use this approach as a tool to get insight into mental models (concerning a topic) of a group of people [4]. The system implements basic organic memory processes [21], hence we call it MnemoWiki, with respect to the Greek goddess of memory - Mnemosyne. This paper will outline some theoretic background knowledge, followed by a description of an application scenario and implementation details.

\section{Theoretical Background}

\subsection{Fading Trails and Items}

Vannevar Bush (1945) predicted technologies including hypertext, Internet, speech recognition and online encyclopedias [3]. In his article he also stated that the human mind operates by association: 'With one item in its grasp, it snaps instantly to the next that is suggested by the association of thoughts, in accordance with some intricate web of trails carried by the cells of the brain. It has other characteristics, of course; trails that are not frequently followed are prone to fade, items are not fully permanent, memory is transitory.' Obviously, he proposed links that fade away and non-permanent items, respectively websites, in order to copy mechanisms of the human memory.

\subsection{Constructing Knowledge}

Piaget described in his theory of cognitive development that the cognitive structures, which we call knowledge, couldn't be understood as a copy of reality, in fact they are the result of adaptation [20]. He further pointed out that cognitive development consists of two components. On the one hand ever changing content and structures and on the other hand unchangeable functions [9] [10] [19].

Piaget also described the relationship of an acting subject (learner) towards an object (learning object) as result of operations (e.g. browsing and editing). The observed attributes of the object direct the mental coordination of the subjects behavior, which in turn changes the impression of the objects attributes and thus changes the subjects behavior again (and so on). This recursive operational loop is described as "coordination" [8] and can be interpreted as constructive observation process. We assume that the visualization of the changing metastructure in MnemoWiki will support this process, by enabling the learner to construct efficient schemes and mental models (as well as revealing them).

\subsection{Mental Modeling}

According to literature there's a broad range of definitions on mental models. It can be a "knowledge structure", which is a collection of connected "mental objects, each with an explicit representation of state, an explicit representation of its topological connections to other objects, and a set of internal parameters" [27]. Further definitions state that "mental models are visually structured propositions that consist of objects and the relationships between objects" [23], they also consist of declarative and procedural knowledge organized by categorization and relations [27]. Mental models help people interpreting signals from the environment, in order to work out appropriate actions [22]. During the process of conceptual change they also continuously reorganize and transform. Thereby, the development of mental models varies as the learner's experience increases [26].

From the view of cognitive psychology, the user has now built efficient schemes that allow automatic and faster processing. If processing takes place in the form of means-end analysis instead (with respect to missing expert schemes), this requires a large amount of cognitive processing capacity [24]. 


\subsection{Graph Theory}

Graph theory can be used for visualization and formalization of structure transformations. The formal definition of a graph depends on its visual appearance [7][2]. For the approach described in this paper, we use and transform a weighted undirected graph. The graph used in this approach is defined as follows:

Let $G=(V, E, \omega, \delta)$ be weighted undirected graph, with a set of vertices $V$, a set of edges $E$, the edge weights represented as the function $\omega: E \rightarrow \mathbf{R}$ that assigns each edge $e \in E$ a weight $\omega(e)$ and let $0<\omega \leq 1$ be a real number. The vertices weight is represented as the function $\delta: V \rightarrow \mathbf{N}$ which assigns each node $v \in V$ a weight $\delta(v)$ and let $0<\delta \leq n$ be a natural number.

Graph transformation is the rule-based manipulation of graphs [2]. The structure of our graph is transformed by continuous weighting of nodes and edges, depending on user 'voting' and traversal history. On the conceptual level, the weight can be interpreted as 'relevance' of contents (with respect to the knowledge domain) and their connections.

\subsection{Information Overload}

The avoidance of loss of information was the motivation for the initial proposal for starting the www as semantic web, by using concept nodes linked from documents and different classes of links [1]. Meanwhile the web was built much easier, without document retrieval mechanism, as every step in the direction of the semantic web turned out to afford useful ontologies and architecture (such as RDF) to support them. Besides, it takes a significant amount of work to add metainformation [17].

Nevertheless, the simple technique still allows a fast growth of the web and the information stored in it. According to netcraft.com, in their automated survey were over 155.230.051 web servers in December 2007 [18]. According to Technorati.com, the number of blogs doubles about every 6 month with a total of 35.3 million blogs as of April 2006 [25].

The new paradigm is that everyone is creating hypermedia content and information for the web by using the web.
The effect is that a learner or a researcher has millions of pieces of information at his fingertips, differing in quality and relevance to the actual task. This may result in the inability to discern between facts because of the large amount of conflicting reports available.

A hypermedia system viewed from an outside position will appear as structured network of $\mathrm{n}: \mathrm{m}$ relations. However, the surfer inside the system experiences $1: \mathrm{m}$ relations (one way in, ' $m$ ' ways out of the page), leaving the task of contextual structuring to him. With an everchanging structure in a Wiki, this might become difficult, since exhaustive repetitive exploration is required.

\section{Oblivion in e-Learning}

As an answer to Information overload we propose the adaptation of consolidation mechanisms as found in the human memory - by letting unused things fade away. The system thereby supports the learner with a consolidated, less complex view of the knowledge base (here a Wiki system). This idea can be derived from the Hebbian theory of synaptic plasticity [5], which is described as time-dependent, local, interactive mechanism that increases synaptic efficiency as a result of pre- and postsynaptic

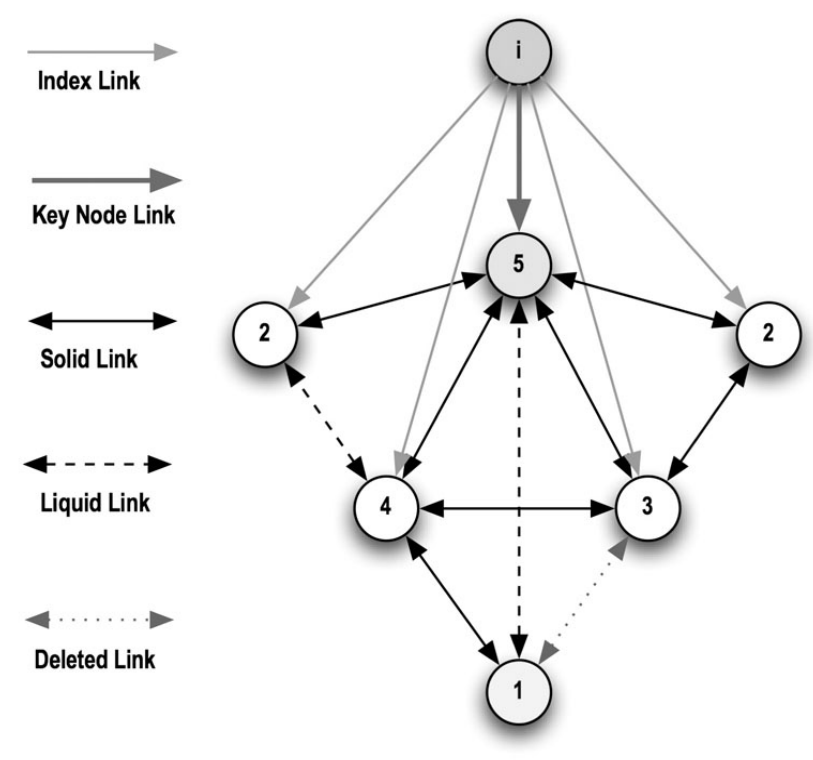

Figure 1. Link and vote scheme. 
activity. We apply this weighting of links to a hypertext knowledge base and use additional weighting of nodes.

Let's assume every link (edge between nodes) in our hypertext system has a predefined lifetime, which increases with use and decreases with time. Naturally, all links, which are used frequently, will be strengthened, while unused links will disappear, leaving a structure composed of the main most used paths. Consequently, a memory system such as this would be completely determined by its usage, which means it could simply forget all links. In order to prevent this, we add a mechanism that discerns between different classes of links.

As you can see in Figure 1, the first classes are "Index Link" and "Keynode Link". The links from the index are directed, as they refer to the metastructure navigation, which is always present and "virtual" to the system itself, e.g. using the tree for the navigation only affects edges (or arcs) between two content nodes.

If a link is added to the system, its class is called "Liquid Link" as we are not sure if this one will prevail. It has a lifetime, which decreases in time. With usage, this lifetime will be increased. Frequent usage can rise the lifetime above a certain threshold and will transform the link to a "Solid Link" class, which simply means that it is approved by the users and gains "immortality". In order to track also the disintegration, the last class is "Deleted Links".

Besides the relevance of the links, we also need to know the relevance of the nodes. Therefore we use a simplified version of the Kleinberg [16] vote algorithm, which was used as a base for the Google kernel. Kleinberg stated that the relevance of a document requires human assessment, which is granted when a person establishes a link (vote) to it.

The algorithm discerns between nodes with many incoming links and nodes with many outgoing links. In order to simplify this, we just treat the links as objects, containing a bidirectional (incoming and outgoing) path. In Figure 1 , the numbers inside the nodes denote this. Counting the links lets us then determine the importance of a node. Thereby, nodes with many links are referred to as key nodes, as they contain important structural information of the knowledge base.
Finally, we visualize the relevant paths (links) and documents (nodes) for the user. We can use color or size for this task, e.g. the most important (supporting) structures are fat lines, less important links are thinner lines, Key nodes are big and bright, less frequently visited nodes are small and grey and so on. Using the weighted links it is easy to implement a mechanism from cognitive psychology, which is called "chain of ideas". Thereby the user gets a suggestion of the most used learning path, based on his actual position. The Algorithm for this is as follows:
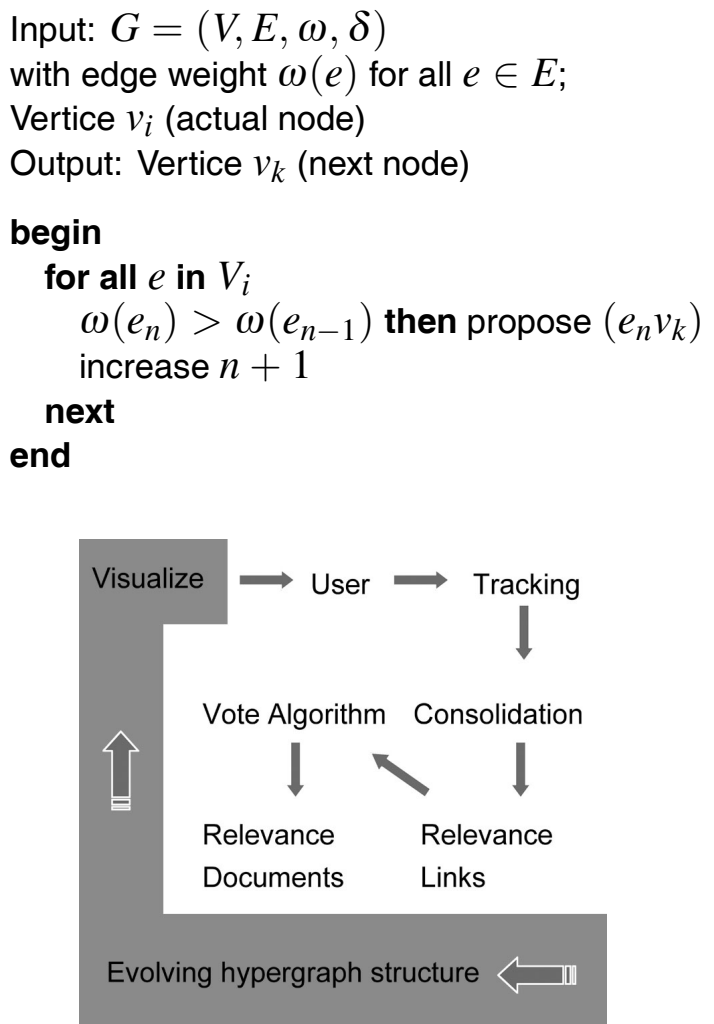

Figure 2. Processing scheme.

Figure 2 describes the processing of information, which is as follows. The user creates Documents and Links, from which the vote algorithm then determines the relevance of the documents. By tracking the user, the system consolidates the links and determines their relevance, which affects again the relevance of the documents. From this data on the relevance of documents and links a hyper graph structure evolves, which is visualized. The visualization can be used by the user as a navigation tool, as well as a view on the main structure of the system for learning purpose. 


\section{Application}

In short, MnemoWiki primarily supports the learner by changing, according to his progress, and secondary, motivates frequent usage due to its "forgetting" nature. This becomes comprehensive given a group of learners where everybody is responsible to create a certain number of documents to feed the knowledge base. The task of the teacher would be meaningful selection of the topics and some guiding feedback on the overall progress. The complexity of the topics must be chosen in a way, allowing an initial independent and parallel directed information search by the learners. Each topic will then be refined in classes, e.g. topics could be well-described methods from a knowledge domain, while the application of these methods is shown in classes or tutorials.

During the process of creation, the learners become aware of the evolving structure and the connections to other topics. This helps them understand the main structure of the knowledge domain while constructing their own view on specific topics from this domain.

Thereby, an important task for the learner is the search for connections from foreign to own articles. As in the end everyone from the group should know most of the created contents (because of the examination), the movement inside the system will further evolve the structure, thus shaping an individual picture of the knowledge of the group. We could also call this the individual mental model of the group concerning this specific domain of knowledge. Several experiments such as this might shape a more generic model. No use of the system instead will disorder (disconnect) the whole information, leaving an unmotivated learning group with nothing than snippets of unconnected information.

\section{Some Implementation Notes}

The visualization of the metastructure is done by a java applet in the browser of the user, which gets its information of the actual structure of the Wiki via an XML-RPC interface (see Figure 3). XML-RPC is a specification and a set of implementations, which allows procedure calls, thereby transmission, processing, and return of complex data structures over the Internet. In

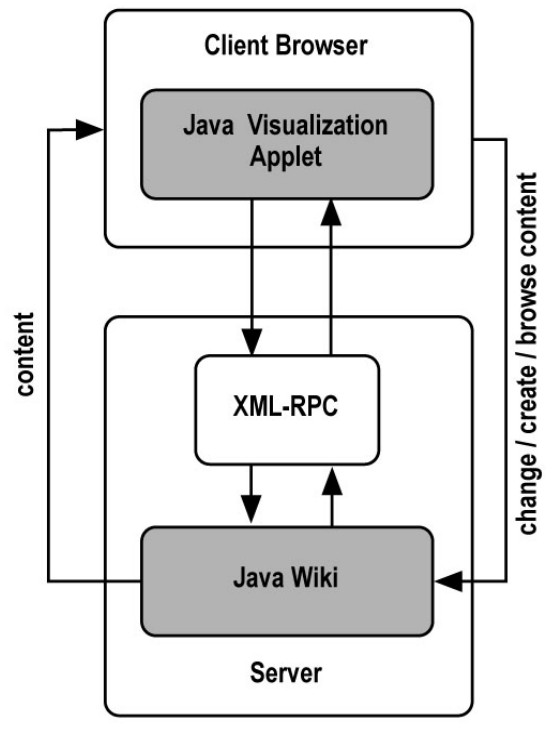

Figure 3. Structure update.

MnemoWiki, XML-RPC gives us the possibility to change the visualization whenever the Wiki Structure is changed.

Each of the nodes in the visualization represents a Wiki page, if there is an edge from node $A$ to node $B$, this means that $A$ is linked to $B$ and vice versa, as all links are bidirectional. If a node $\mathrm{C}$ is not connected to any other nodes, it will not be displayed, however, it remains in the system. When a node is clicked all links connected to this node are read from the Wiki and rendered.

\section{Conclusions and Further Work}

Facing the challenge of growing information, with a special attention to the acceleration of this process due to web 2.0 technologies and the according philosophy, we've shown that a basic memory consolidation mechanism can deal with the problem of information overload. We explained the application of this mechanism to a hypertext system, in this case a Wiki, which is meant for e-learning purpose. Although the application in a learning environment has been discussed here, it is possible to implement this also in other knowledge systems and web 2.0 applications if needed. The visualization grants an overview of the key structures of the knowledge system, thereby enabling the user to gain 
a comprehensive view of the contents, further it is used for navigation purpose. However, the intention of this approach is not only a filtered visual view of the system to give the user a better insight, rather we affect the structure of the hypertext system itself, by applying rules for self organization. The structure evolves according to its use, providing the aspects of information which are used most and thus highly relevant.

Additionally we propose this kind of system as a tool for studying mental models. In the described MnemoWiki the result would be individual mental models of groups concerning a special knowledge domain. Aggregation of different groups should produce more generic models, which might then be applied in usability studies (user expectations) or even in design processes as in-depth target group analysis.

Our future work will focus on the conduction of experiments with the described MnemoWiki system in e-learning contexts. So far our driving questions are: How does the revelation of key structures contribute towards understanding a complex knowledge domain? How does the consolidation of information support human cognitive performance? Regarding the future of the web, with respect to the intelligent web, consolidation methods will be needed to prevent information overload.

\section{References}

[1] T. BERNERS-LEE, Information Management: A Proposal. W3 Archive; 1989. http://www.w3.org/ History/1989/proposal.html [02/28/2008]

[2] U. BRANDES AND T. ERLEBACH editors. Fundamentals. In: Network Analysis - Methodo-logical Foundations. Lecture Notes in Computer Science 2005, 3418: pp. 7-15.

[3] V. Bush, As We May Think. Boston: Atlantic monthly press; 1945.

[4] X. Chen, e.a. Discovering Conceptual Page Hierarchy of a Web Site from User Traversal History. Lecture Notes in Computer Science 2005, 3584: pp. 536-543.

[5] M. DeHMER, e.a. Measuring the Structural Similarity of Web-based Documents: A Novel Approach. International Journal of Compu-tational Intelligence 2006; 3 (1): pp. 1-7.
[6] M. Ebner, A. Holzinger And H. Maurer, Web 2.0 Technology: Future Interfaces for Tech-nology Enhanced Learning? Lecture Notes in Computer Science 2007, 4556: pp. 559-568.

[7] R. DIESTEL, Graph Theory. Heidelberg: SpringerVerlag; 2005.

[8] H. Foerster, v. Objects: Tokens for (Eigen-) Behaviors, Observing Systems. Seaside: Intersystems; 1981.

[9] E. Glasersfeld, v. Aspects of Radical Constructivism and its Educational Recom-mandations. Proceedings of the ICME-7; 1992 Aug 17-23; Quebec, Canada.

[10] E. GLASERSFELD, v. Constructivism reconstruct-ed: A reply to suchting. Science and Education 1992; 1(4): pp. 379-384.

[11] D. HEBB, The Organization of Behavior: a neuropsychological approach. New York: Wiley; 1949.

[12] A. Holzinger, M. KicKmeier-Rust And D. ALBERT, Dynamic Media in Computer Science Education; Content Complexity and Learning Performance: Is Less More? Educational Technology \& Society 2008, 11(1): pp. 279-290.

[13] A. Holzinger, A. K. Nischelwitzer AND M. MeIsenberger, Mobile Phones as a Challenge for m-Learning. Examples for Mobile Interactive Learning Objects (MILOs). IEEE PerCom 05;2005; Kauai Island, Hawai. pp. 307-311.

[14] A. Holzinger, A. K. Nischelwitzer AND M. MEISENBERGER, Lifelong-learning Support by Mlearning: Example Scenarios. ACM eLearn Magazine 2005; 5:12.

[15] A. Holzinger, A. K. Nischelwitzer AND M. D. KICKMEIER-RUST, Pervasive E-Education sup-ports Life Long Learning: Some Examples of X-Media Learning Objects; 2006. Online available:[05/05/2008] http: //www . wccee2006.org/papers/445.pdf

[16] J. KLEINBERG, Authoritative sources in a hyperlinked environment. Journal of the ACM (JACM) 1999; 46(5): pp. 604-632.

[17] H. Maurer, Web - Based Knowledge Management. IEEE Computer 1998; 31(3): pp. 122-123.

[18] Netcraft.com Dec. 2007 Web Server Survey. Netcraft.com; 2007. http://news.netcraft. com/archives/2007/12/29/ december_2007 _web_ server_survey.html [02/28/2008]

[19] P. MussEN, editor. Piaget's theory. Handbook of child psychology. New York: Wiley; 1997.

[20] J. Piaget, On the development of memory and identity. Worchester (MA): Clark University Press; 1961. 
[21] S. Ribeiro, D. Gervasoni, E. S. SoARes, Y. Zhou, S. C. LIN, ET AL. Long-lasting Novelty-induced Neuronal Reverberation during Slow-wave Sleep in Multiple Forebrain Areas. PLoS Biology 2004; 2(1): pp. 126-137.

[22] Schnotz, W., Preub, A. Task - dependent construction of mental models as a basis for conceptual change. European Journal of Psychology Education 1997; 12(2): pp. 185-211.

[23] N. Staggers, A. F. Norcio, Mental models: concepts for human-computer interaction research. International Journal of Man-Machine Studies 1993; 38 (4): pp. 587-605.

[24] J. SWELLER, Evolution of human cognitive architecture. The Psychology of Learning and Motivation. San Diego: Academic Press; 2003.

[25] D. SIFRY, On Blogosphere Growth. Technorati; 2006.http://technorati.com/weblog/2006/ 02/81.html [02/28/2008]

[26] J. R. VELDOF, K. BEAVER, Going mental: tackling mental models for the online library tu-torial at the University of Minnesota libraries. Research Strategies 2001; 18(1): pp. 3-20.

[27] M. D. WiLliams, Human reasoning about a simple physical system. Mental Models. New York: Lawrence Erlbaum Associates; 1983.

[28] L. A. WOZNY, The application of metaphor, analogy and conceptual models in computer systems. Interacting with Computers 1989; 1(3): pp. 273-283.

Received: June, 2008 Accepted: September, 2008

Contact addresses:

Christian Stickel

Social Learning

Graz University of Technology Steyrergasse 30/I, A-8010 Graz stickel@tugraz.at

Martin Ebner Social Learning Graz University of Technology Steyrergasse 30/I, A-8010 Graz martin.ebner@tugraz.at

Andreas Holzinger Research Unit HCI4MED

Institute of Medical Informatics, Statistics and Documentation

Medical University Graz Auenbruggerplatz 2/V, A-8036 Graz andreas.holzinger@meduni-graz . at
Christian Stickel is currently writing his Ph.d. Thesis on "Cognitive Performance Support in Information Systems". He s working as technican in the Department for Social Learning of Computer and Information Services at Graz University of Technology and part of the Research Unit HCI4MED. He is head of the expert commitee "Usability \& E-Learning" of the German Internet Association. Christian Stickel holds a diploma in information technology from the University of Applied Sciences Frankfurt/Main. For almost 10 years, he worked as multimedia designer, developer and consultant for several advertisement agencies. His research interest is in cognitive ergonomics, which is concerned with mental processes, information visualization, neuropsychology, medical hypnosis and overall HCI \& UE.

MARTIN EBNER is currently head of the Department for Social Learning of Computer and Information Services at Graz University of Technology. He is responsible for all e-Learning activities of the University. His research focuses strongly on the use of Web 2.0 for teaching and learning purposes. Martin has delivered a number of lectures and seminars around the topic of e-Learning and the use of computers in educational settings. He studied civil engineering from 1995-2000 and got his M.Sc. from the Institute of Structural Concrete. Afterwards Martin worked as scientific assistant at the Institute of Structural Concrete and wrote his $\mathrm{Ph}$.D. thesis about e-Learning in structural engineering. Since 2005 he holds a Ph.D. in technical sciences from Graz University of Technology. From 2005 to 2006 he worked at the Institute for Building Informatics as Assistant Professor. Since September 2006 Martin is head of the Department for Social Learning at the Computing and Information Services. He wrote not only an amount of international publications and gave a number of presentations about e-Learning, he is also member of various national and international research groups and scientific boards. Martin is one of the biggest EduBlogger in the German speaking area and conducts the e-Learning Blog (http://elearningblog.tugraz.at).

ANDREAS HOLZINGER is head of the Research Unit HCI4MED, Institute of Medical Informatics, Statistics \& Documentation (IMI), Medical University Graz; Associate Professor at Graz University of Technology and chair of the Workgroup Human-Computer Interaction and Usability Engineering (HCI\&UE) of the Austrian Computer Society. Andreas holds M.Sc. in physics and psychology, MPh in media pedagogy and sociology and a Ph.D. in cognitive science from Graz University. He got his second doctorate in applied information processing from Graz University of Technology. He was Visiting Lecturer at the Nations Health Career Center, Berlin (Germany) in 2002 and 2003, Visiting Professor at Innsbruck University, Institute for Organization \& Learning in 2004/2005, Visiting Professor at Vienna University of Technology, Institute for Software Technology \& Interactive Systems in 2005/2006, Visiting Professor at Vienna University of Economics, Health Care Management in 2006/2007 and Visiting Professor at Middlesex University London, School of Computing Science in 2007. His current research areas include technology-enhanced life long learning, gerontechnology and accessibility. He has served as consultant for several European ministries, industry and as national expert in the European Commission initiative Europe: towards an Information Society for all. He is member of the ACM, IEEE, BCS, German Society of Psychology and board member of the Austrian Computer Society (OCG). 
